Correction

\title{
Correction: Wang, M. and Yin, S. Some Liouville Theorems on Finsler Manifolds. Mathematics, 2019, 7,351
}

\author{
Songting Yin ${ }^{1, *}$ and Minqiu Wang ${ }^{2}$ \\ 1 Department of Mathematics and Computer Science, Tongling University, Tongling 244000, China \\ 2 Department of Mathematics and Physics, Hefei University, Hefei 230601, China \\ * Correspondence: yst419@163.com
}

Received: 21 June 2019; Accepted: 25 June 2019; Published: 26 June 2019

The authors are sorry to report that the proof of case III of Theorem 1.2 in their recently published paper [1] was incorrect. Upon revising the manuscript, they mistakenly thought that $u_{k}$ is in $H^{2}$ and $\Delta u_{k}=0$ holds on some particular subset of the manifold $M$. Consequently, the authors wish to make the following corrections to the paper at this time:

We first remark that the proof of case III is different from the one in [2] because there is a mistake there. For every $k \in \mathbb{R}^{+}$, set

$$
u_{k}= \begin{cases}k, & u \geq k \\ u, & u<k .\end{cases}
$$

In what follows, we will follow the arguments in [3] (p. 178) with some modifications. Let $\beta$ be a symmetric, convex, and bounded smooth function with $\left|\beta^{\prime}\right|<1$ and $|s|<\beta<\epsilon+|s|$, where $0<\epsilon<1$ is such that $u-\epsilon>0$. Define

$$
\tilde{u}_{k}=\frac{u+k}{2}-\frac{\beta(u-k)}{2} .
$$

Then, for any positive integer $k$, it holds that $\tilde{u}_{k}>\frac{u+k}{2}-\frac{\epsilon+|u-k|}{2}>0$. Moreover, $\tilde{u}_{k}$ is a superharmonic function in a weak sense. Indeed, by definition, we have $d \tilde{u}_{k}=\frac{1}{2}\left(1-\beta^{\prime}\right) d u$, which yields $\nabla \tilde{u}_{k}=\frac{1}{2}\left(1-\beta^{\prime}\right) \nabla u$ by Legendre transformation. As $\tilde{u}_{k} \in H_{l o c}^{2}$ and thus $\Delta \tilde{u}_{k}=0$ a.e. on $M \backslash M_{u}$, for $\psi$ defined in Case I, we have

$$
\begin{aligned}
2 \int_{B_{x_{0}}^{-}(R)} \psi \Delta \tilde{u}_{k} d \mu & =-2 \int_{B_{x_{0}}^{-}(R)} d \psi\left(\nabla \tilde{u}_{k}\right) d \mu=-\int_{B_{x_{0}}^{-}(R)}\left(1-\beta^{\prime}\right) d \psi(\nabla u) d \mu \\
& =-\int_{B_{x_{0}}^{-}(R)} d\left[\left(1-\beta^{\prime}\right) \psi\right](\nabla u) d \mu-\int_{B_{\bar{x}_{0}}^{-}(R)} \beta^{\prime \prime} \psi F(\nabla u)^{2} d \mu \\
& \leq-\int_{B_{x_{0}}^{-}(R)} d\left[\left(1-\beta^{\prime}\right) \psi\right](\nabla u) d \mu=\int_{B_{x_{0}}^{-}(R)}\left(1-\beta^{\prime}\right) \psi \Delta u d \mu \\
& \leq 0 .
\end{aligned}
$$

The last step holds because $\left(1-\beta^{\prime}\right) \psi$ is differentiable almost everywhere on $B_{x_{0}}^{-}(R)$ with bounded differential, and $u$ is superharmonic. Moreover, $\tilde{u}_{k}$ is smooth on the open subset $M_{u}$ and is also superharmonic, in the classical sense, on $M_{u}$. Notice that $\psi$ is differentiable almost everywhere on 
$B_{x_{0}}^{-}(R)$ with bounded differential. Hence, by similar arguments, we can also obtain (4) (see [1], p. 6) for $\tilde{u}_{k}$ on $B_{x_{0}}^{-}(R)$ as in case I. Set $v_{k}=\tilde{u}_{k}^{\frac{q}{2}}$ for any $q \in(0,1)$. Then we have (5) (see [1], p. 7) as follows:

$$
\begin{aligned}
& \left(1-\frac{1}{q}\right)^{2} \int_{B_{x_{0}}^{-}(R)} \psi^{2} F\left(\nabla v_{k}\right)^{2} d \mu \\
\leq & \widehat{C}\left(\int_{B_{x_{0}}^{-}(R) \backslash \bar{B}_{x_{0}}^{-}\left(r_{0}\right)} v_{k}^{2}\right)^{\frac{1}{2}}\left(\left(1-\frac{1}{q}\right)^{2} \int_{B_{x_{0}}^{-}(R) \backslash \bar{B}_{x_{0}}^{-}\left(r_{0}\right)} \psi^{2} F\left(\nabla v_{k}\right)^{2} d \mu\right)^{\frac{1}{2}} \\
= & \widehat{C}\left(V_{q}(R)-V_{q}\left(r_{0}\right)\right)^{\frac{1}{2}}\left(\left(1-\frac{1}{q}\right)^{2} \int_{B_{x_{0}}^{-}(R) \backslash \bar{B}_{x_{0}}^{-}\left(r_{0}\right)} \psi^{2} F\left(\nabla v_{k}\right)^{2} d \mu\right)^{\frac{1}{2}} .
\end{aligned}
$$

On the other hand, note that $\tilde{u}_{k} \leq k$, and thus

$$
\int_{B_{x_{0}}^{-}(R)} \tilde{u}_{k}^{q} d \mu \leq \int_{B_{x_{0}}^{-}(R)} k^{q} d \mu=k^{q} V(R),
$$

which implies that

$$
\int_{1}^{\infty} \frac{r}{V_{q}(r)} d r=\infty
$$

Then by the same discussion in the proof of (2) (see [1], pp. 5-7) and Case I of (1) (see [1], p. 7), we show that this $\tilde{u}_{k}$ is constant. Take then a sequence $\beta_{n}$ (such that each $\beta_{n}$ satisfies the same properties as $\beta$ ) uniformly converging to the absolute value function. Every $\tilde{u}_{k, n}$ is then constant. These constants are bounded (they are in $(0, k)$ ). Thus, up to pass to a subsequence $\tilde{u}_{k, n}$ converges uniformly to $u_{k}$ and to a constant at the same time. Hence, $u_{k}$ must be constant. $k$ being arbitrary, $u$ is also constant.

Funding: This project is supported by TLXYXM (No. 2018tlxyzd02) and EYTVSP (No.gxfx2017095).

Acknowledgments: The authors would like to sincerely thank the Academic Editor, Erasmo Caponio for all the work done.

Conflicts of Interest: The authors declare no conflict of interest.

\section{References}

1. Wang, M.; Yin, S. Some Liouville theorems on Finsler manifolds. Mathematics 2019, 7, 351. [CrossRef]

2. Zhang, F.; Xia, Q. Some Liouville-type theorems for harmonic functions on Finsler manifolds. J. Math. Anal. Appl. 2014, 417, 979-995. [CrossRef]

3. Grigor'yan, A. Analytic and geometric background of recurrence and nonexplosion of the brownian motion on Riemannian manifolds. Bull. Am. Math. Soc. (N.S.) 1999, 36, 135-249. [CrossRef]

(c) 2019 by the authors. Licensee MDPI, Basel, Switzerland. This article is an open access article distributed under the terms and conditions of the Creative Commons Attribution (CC BY) license (http:/ / creativecommons.org/licenses/by/4.0/). 